\title{
Ninfa e Gradiva: dalla percezione individuale alla memoria storica sovrapersonale
}

Nymphe et Gradiva: de la perception individuelle à la mémoire historique superpersonnelle

Nymph and Gradiva: From the Individual Perception to the Historical and Transpersonal Memory

\section{Daniela Sacco}

\section{(2) OpenEdition}

\section{Journals}

Edizione digitale

URL: http://journals.openedition.org/cei/3080

DOI: $10.4000 /$ cei.3080

ISSN: 2260-779X

\section{Editore}

UGA Éditions/Université Grenoble Alpes

\section{Edizione cartacea}

Data di pubblicazione: 30 décembre 2016

Paginazione: $45-60$

ISBN: 978-2-84310-337-7

ISSN: 1770-9571

Notizia bibliografica digitale

Daniela Sacco, «Ninfa e Gradiva: dalla percezione individuale alla memoria storica sovrapersonale», Cahiers d'études italiennes [Online], 23 | 2016, online dal 23 janvier 2017, consultato il 26 mars 2021. URL: http://journals.openedition.org/cei/3080 ; DOI: https://doi.org/10.4000/cei.3080 


\title{
NINFA E GRADIVA: DALLA PERCEZIONE INDIVIDUALE ALLA MEMORIA STORICA SOVRAPERSONALE
}

\author{
Daniela Sacco \\ Università IUAV di Venezia
}

Le riflessioni di André Jolles sulla ninfa, contenute nel carteggio fittizio con Aby Warburg (1900) ${ }^{\mathrm{I}}$ sulla figura femminile incedente ritratta nella Nascita di San Giovanni Battista del Ghirlandaio, sono particolarmente eloquenti rispetto alle speculazioni relative al mito sia precedenti che successive il carteggio, se confrontate con il racconto di Wilhelm Jensen, Gradiva, una fantasia pompeiana (1903), e anche rispetto all'interesse mostrato dalla psicoanalisi nel testo di Sigmund Freud Delirio e sogni della "Gradiva» di Wilhelm Jensen (1906). È proprio la convergenza d'interesse da parte delle scienze nascenti nei primi del Novecento, la (nuova) scienza dell'arte e la psicoanalisi, per la medesima figura, a risultare particolarmente significativa per le riflessioni sul mito. La ninfa è infatti una figura eccentrica e trasversale ${ }^{2}$, che attraversa discipline differenti riproducendo la stessa trasversalità della materia mitica.

La fortuna degli studi warburghiani, che ha riconosciuto nella ninfa una figura teorica fondamentale, ha indotto alcuni a riportare i contenuti espressi da Jolles nel carteggio, concentrati nella Epistula prima, al pensiero dello storico dell'arte tedesco. Silvia Contarini ${ }^{3}$ ha per esempio

I. A. Warburg, Ninfa fiorentina Igoo, Warburg Institute Archive: WIA III, II8, trad. it. La ninfa: uno scambio di lettere tra A. Jolles e A. Warburg, in A. Warburg, Opere, vol. I, M. Ghelardi (a cura di), Torino, Aragno, 2004, pp. 243-255. Per un primo inquadramento del testo si veda anche S. Contarini, M. Ghelardi, "Die verkörperte Bewegung": la ninfa, "Aut Aut», 32I-322, maggio-agosto 2004, pp. 32-45.

2. Cfr. G. Didi-Huberman, Il passo leggero dell'ancella. Sul sapere eccentrico delle immagini, trad. it. di F. Massa, Bologna, EDB, 2015 .

3. Cfr. S. Contarini, "Botticelli ritrovato": frammenti di dialogo tra Aby Warburg e André Jolles, "Prospettiva", 68, 1992, pp. 87-93; Nello specchio di van Eyck. Warburg, Jolles, Huizinga, «Intersezioni», 2, 200I, pp. 30I-334; Tra folklore e scienza dell'arte: le forme del mito in André Jolles, in M. Bertozzi (a cura di), Aby Warburg e le metamorfosi degli antichi dei, Modena, Panini, 2002, pp. I53-I72. 
individuato, negli scritti giovanili precedenti il carteggio, il debito di Jolles nei confronti di Warburg riguardo all'idea fondante del movimento come manifestazione di pathos e come cifra della riattivazione dell'antichità classica in epoca rinascimentale: un'idea argomentata in quello stesso periodo da Warburg ne "La nascita di Venere" e la "Primavera" di Sandro Botticelli (I893) e I costumi teatrali per gli intermezzi del I589 (I895). Il riconoscimento dell'importanza dell'elemento emozionale all'interno della rappresentazione artistica si accompagna in Warburg alla consapevolezza del valore dell'approccio psicologico alla ricerca storico-artistica. Le implicazioni psicologiche dello studio del movimento nel contesto artistico rinascimentale sembrano essere proprio l'apporto warburghiano rispetto a quanto Leon Battista Alberti aveva già individuato come cifra dell'antico nel Rinascimento. Un apporto che Jolles fa proprio nel proporre un metodo della scienza dell'arte fondato «su leggi psicologiche» che legano assieme "Arte, Linguaggio, Mito e Cultura» 4 .

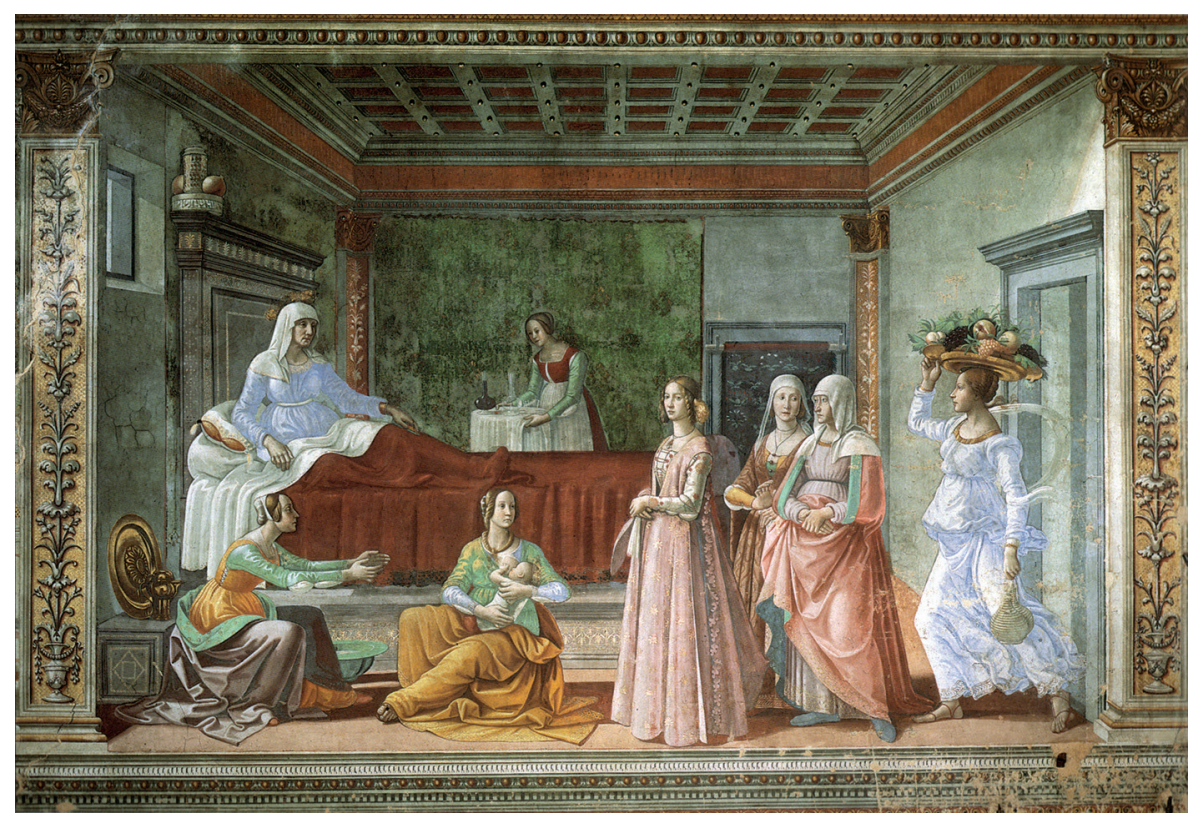

Fig. I. - Domenico Ghirlandaio, Nascita di Giovanni Battista (I485). Firenze, Santa Maria Novella, Cappella Tornabuoni. 
L'importanza attribuita da Jolles al valore psicologico dei fenomeni artistici impedisce di credere che la divisione di ruoli giocata nel carteggio dai due studiosi, Jolles nei panni dell'esteta e Warburg del filologo, corrisponda a realtà. Come è stato già rilevatos, l'approccio giovanile estetizzante era in realtà già superato alla data del carteggio da Jolles, che in quell'occasione partecipava a un serio ludere in cui l'oggetto del disquisire era il destino di una scienza nascente, $\mathrm{e}$ in cui profondità storico-filologica e ricerca di senso hanno la stessa fondamentale importanza. Come ha ben osservato Salvatore Settis ${ }^{6}$, il lusus della ninfa è un gioco di ruoli in cui la dialettica ha finalità maieutiche.

\section{Due ninfe a confronto}

Le caratteristiche con cui Jolles descrive la ninfa nella Epistula prima, datata 23 dicembre 1900, possono essere individuate nel confronto con quelle descritte nella novella di Jensen commentata da Freud. Gradiva, una fantasia pompeiana fu pubblicata in forma integrale nel $1903^{7}$, ma la questione della sua possibile derivazione dal carteggio Jolles-Warburg, per quanto apparentemente legittima, non è da porre. Sia perché la prima, parziale pubblicazione del carteggio avviene molto più tardi, nel $1970^{8}$, a opera di Gombrich, sia perché, come dichiara Jensen in risposta alle domande di Freud (da un lato interessato a verificare la conoscenza della teoria psicoanalitica da parte dello scrittore tedesco e dall'altro a caccia di tracce autobiografiche che confermassero la sua interpretazione), lo stimolo della scrittura nasce esclusivamente da un'esperienza del tutto simile a quella del protagonista del racconto, ossia la visione del bassorilievo che ritraeva una figura femminile incedente ${ }^{9}$. La risposta di Jensen conferma che l'esperienza legata alla visione della figura incedente o ninfa è un topos, un motivo ricorrente, o piuttosto, come l'ha definita Andrea Pinotti ${ }^{\mathrm{IO}}$, una

5. S. Contarini, Introduzione, in A. Jolles, I travestimenti della letteratura. Saggi critici e teorici (I897-I932), S. Contarini (a cura di), Milano, Bruno Mondadori, 2003, p. xv.

6. Cfr. S. Settis, Presentazione, in J. Seznec, La sopravvivenza degli antichi dei. Saggio sul ruolo della tradizione mitologica nella cultura e nell'arte rinascimentali, trad. it. di G. Niccoli, P. Gonnelli Niccoli, Torino, Bollati Boringhieri, I992, pp. vII-XxIx.

7. Una prima pubblicazione, a puntate, nella rivista viennese «Neue Freie Presse» avviene tra giugno e luglio del 1902 .

8. Cfr. E. Gombrich, Aby Warburg. An Intellectual Biography, London, The Warburg Institute, I970.

9. S. Freud, Saggi sull'arte, la letteratura e il linguaggio, trad. it. di S. Daniele et al., Torino, Bollati Boringhieri, I99I, vol. 2, pp. 25I sg.

Io. A. Pinotti, Ninfa tra Eidos e formula, aspetti fenomenologici nell'iconologia di Warburg, in M. Ophälders (a cura di), Fenomenologie e arte. Immagini e figure riflesse nella filosofia, Milano, Mimesis, 2005, pp. I39-I48. 
sorta di Ur-Nympha, che ha accomunato, attraverso epoche e ambiti disciplinari differenti, molti autori. E se la fantasia di Jensen, comune a quella di Jolles, può essere ricondotta tout court alla fantasia maschile vittoriana di fine Ottocento, dove la ninfa è incarnazione del desiderio erotico, è possibile procedere a ritroso nel tempo per monitorarne l'apparizione e andare più in profondità nell'analisi del suo significato, che appartiene alla tradizione classica.

Sono molte le analogie tra lo scritto di Jolles e quello di Jensen ${ }^{\text {II; }}$ p prima tra tutte l'identificazione della figura con il movimento. Per Jolles la figura femminile "corre, anzi vola, o meglio si libra»; ha un "modo di camminare leggero e vivace e al contempo molto movimentato»; il suo è un "energico incedere a lunghi passi», "fluttuante», che ha "un'elasticità ondeggiante»; la ninfa è colei che "porta vita e movimento alla scena» di per sé statica e austera. L'oggetto che cattura l'attenzione di Norbert Hanold, il giovane filologo-archeologo protagonista della novella di Jensen, è una figura femminile immortalata "nel suo incedere» in un bassorilievo conservato ai Musei Vaticani; sembra scolpita dall'artista nella «viva impressione del momento in cui ella gli era passata davanti»; appare infusa di vita e questa impressione dipende in primo luogo dal «movimento in cui ella era stata raffigurata». Un movimento che esprime «estrema levità nell'incedere» e «simultanea stasi in se stessa»: una grazia data dalla «aerea fluttuazione e il sicuro appoggio sul terreno». Il movimento è così identificativo della figura che il protagonista nomina "Gradiva», ossia "colei che avanza». Si scoprirà poi che il nome effettivo della fanciulla, il corrispettivo reale dell'immagine del bassorilievo, è Zoe Bertgang, dove Zoe, dal greco, traduce la parola «vita», e Bertgang, nell'etimologia germanica, indica «colei che risplende nel cammino». Quindi per entrambi gli autori, Jolles e Jensen, il movimento è indicativo di vita.

Un altro elemento comune è l'aspetto fantasmatico accompagnato alla figura reale, che è allo stesso tempo oggetto di un delirio. Per Jolles essa è l'oggetto dei sogni che assume «le dimensioni di un affascinante incubo»: è una "figura fantastica» che appare come qualcosa di "sovraterreno", «una dea». Lo stato d'animo che accompagna il pensiero di lei «oscilla tra l'incubo e la favola» e la sensazione è quella di aver "perso la ragione». Nel racconto di Jensen, Gradiva compare al protagonista nell'incubo che

II. Le citazioni testuali che seguono sono tratte da A. Jolles, A. Warburg, La ninfa: uno scambio di lettere [1900], trad. it. di M. Ghelardi, "Aut Aut», 32I-322, maggio-agosto 2004, pp. 46-49, e W. Jensen, Gradiva, una fantasia pompeiana, in W. Jensen, S. Freud, Gradiva, R. Oriani (a cura di), Pordenone, Edizioni Studio Tesi, I992, pp. 5-IOI. 
la vede a Pompei il giorno dell'eruzione del Vesuvio. Da sveglio, invece, la ricerca di lei nei luoghi della realtà è accompagnata da un fantasticare descritto come "uno stato di sogno, più o meno a cavallo tra la piena coscienza e la perdita dei sensi»; è la "condizione dell'anima che chiamiamo sogno» a portare il protagonista «indietro nel tempo, a Pompei». L'alternarsi tra reale e fantastico, razionale e follia, veglia e sogno, aumenta esponenzialmente fino all'epilogo, che si risolve per il protagonista con il «ritrovato pieno possesso delle facoltà razionali» e una «ritrovata salute mentale».

Sia Jolles che Jensen si interrogano sulle origini greche della figura, il primo ipotizzando la discendenza da un «nobile lignaggio greco», il secondo riconoscendo l'"origine ellenica» nei tratti del volto "di tipo greco». In entrambi i casi, nella razionalità o nella follia, l'esperienza vissuta a seguito dell'incontro con la fanciulla è l'innamoramento: Jolles, che si riconosce «innamorato perdutamente», è «vittima» di un "flirt intellettuale»; Norbert Hanold, mosso dalla passione, ne insegue le tracce fino a incontrarla in carne e ossa e a suggellarne il ritrovamento con il matrimonio. L'innamoramento inoltre si accompagna in entrambi i casi all'impressione di aver conosciuto precedentemente l'oggetto del desiderio; Jolles si chiede dove ha già visto la fanciulla, con la sensazione che desiderante e desiderato fossero legati da «una conoscenza precedente». L'impressione, scrive Jolles, è di ritrovare un caro amico, un luogo a cui era stato legato in un'epoca precedente. Norbert, a cui il viso di Gradiva «appariva estraneo e tuttavia familiare, quasi l'avesse già visto o se lo fosse già immaginato", scoprirà che la fanciulla era effettivamente una reale conoscenza, risalente all'infanzia. A tutto ciò si accompagna, per entrambi, la sensazione di vedere l'immagine ovunque, in modo quasi ossessivo, riprodotta e mutata in contesti differenti, ma pur sempre riconoscibile. Jolles la vede «in continuazione: sempre diversa e sempre in luoghi difformi»; la riconosce in Salomè, in Giuditta, nel fanciullo Tobia, in un serafino, nell'angelo Gabriele (le figure che Warburg identificherà come variazioni di una stessa Pathosformel). Allo stesso modo Norbert, complice il sogno che lo riporta a vicende mitiche legate ai luoghi di Pompei, assiste a «meravigliose metamorfosi ovidiane» che si compiono nella notte attorno al suo letto. In entrambi i testi, la domanda sull'origine e la provenienza dell'immagine innerva la tensione che dà corpo alle parole di Jolles e muove l'azione della vicenda del protagonista di Jensen. L'identificazione più eloquente di questa figura è esplicitata da Warburg, che nel carteggio la definisce «una dea pagana in esilio»e 
uno «spirito elementare», rivelandone la parentela con Gli dei in esilio (I853) e Gli spiriti elementari (I837) di Heinrich Heine ${ }^{\mathrm{I} 2}$, oltre che con Paracelso ${ }^{\mathrm{I} 3}$.

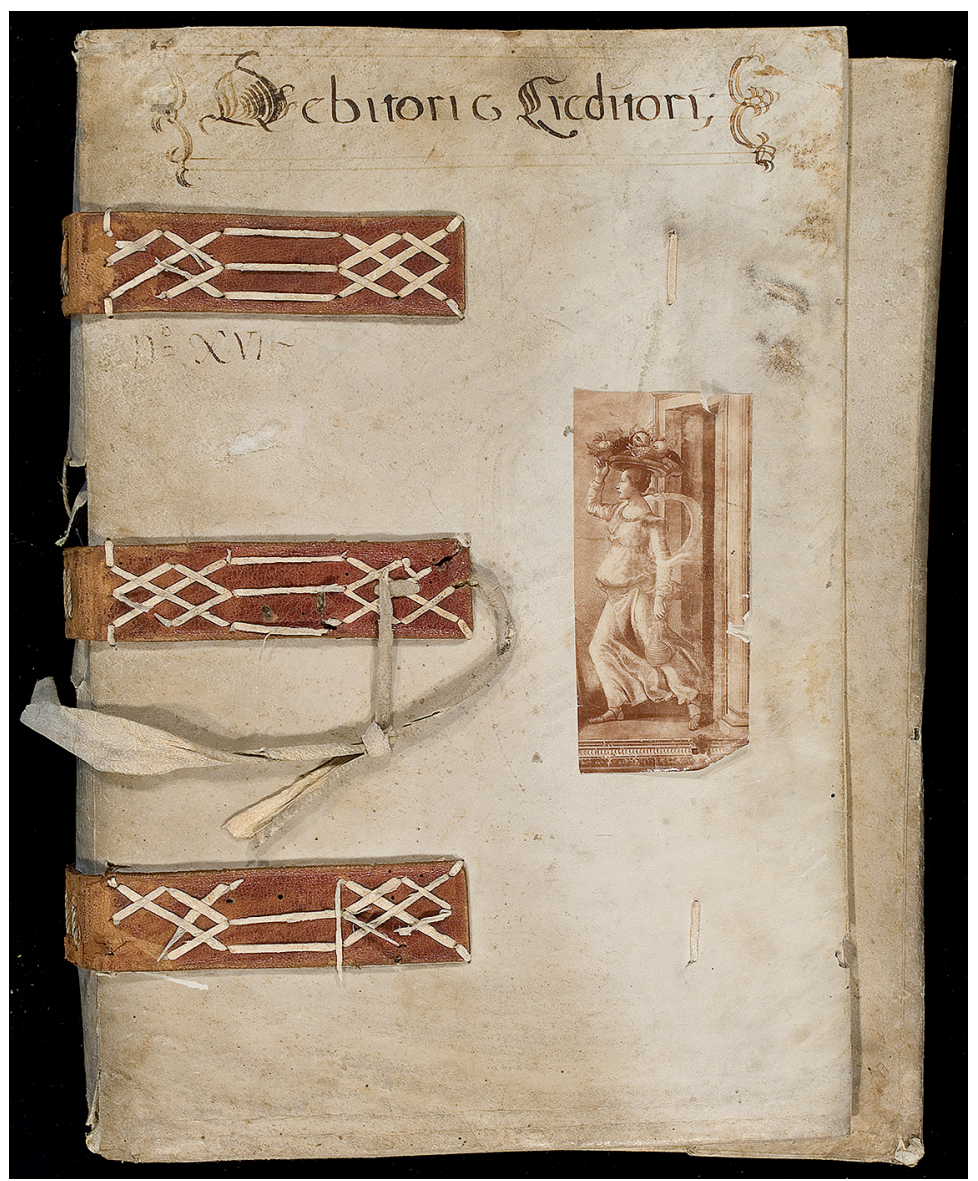

Fig. 2. - Aby Warburg, Nymphenfragment (1986-1900), copertina del manoscritto.

London, Warburg Institute Archive.

I2. Cfr. S. Weigel, Aby Warburgs "Göttin im Exil». Das "Nymphenfragment» zwischen Brief und Taxonomie, gelesen mit Heinrich Heine, in Vorträge aus dem Warburg-Haus, vol. 4, Berlin, De Gruyter, 2000, pp. 65-I03.

13. Cfr. G. Agamben, Nymphae, "Aut Aut», 32I-322, maggio-agosto 2004, pp. 53-67. 
La condizione di esilio (ma si potrebbe utilizzare anche il termine psicoanalitico «rimozione» ${ }^{\mathrm{I}}$ ) della divinità pagana è infatti l'elemento fondamentale dell'interesse per questa figura, che accomuna, nel passaggio tra Otto e Novecento, sia gli studi storico-artistici che quelli della nascente psicologia del profondo, o scienza dell'anima. A essere esiliato o rimosso è propriamente il nucleo originario classico, il paganesimo incarnato dalla ninfa. La figura della ninfa interessa quindi la nuova scienza dell'arte in quanto veicolo della classicità pagana, e la neonata psicoanalisi in quanto figura simbolo dell'anima, anch'essa veicolo del paganesimo.

\section{Ninfa come anima}

Lidentificazione ninfa-anima avviene soprattutto in epoca rinascimentale, ${ }^{\text {Is }}$ nel contesto della tradizione letteraria e figurativa, per influenza della filosofia di ascendenza neoplatonica, che, rielaborando gli elementi costitutivi dell'anima di provenienza platonica e aristotelica, li associa alle figure delle ninfe classiche: le divinità minori, con fattezze di giovani fanciulle, che rappresentano il manifestarsi della natura in acque, monti e boschi, sono da sempre collegate all'acqua che dona e risveglia la vita. La ninfa, designando la donna in età da marito, la fidanzata e giovane sposa, è simbolo dell'anima che scende nella generazione per unirsi al corpo. $\mathrm{Si}$ tratta del dramma della discesa cosmica dell'anima nel mondo attraverso la generazione e della sua risalita al divino, rappresentato esemplarmente ne L'antro delle ninfe di Porfirio, riscoperto dai platonici fiorentini.

Se per Eschilo le ninfe sono donatrici di vita, a partire da Platone, e poi con Aristotele, vita e movimento vengono a coincidere nell'anima: nel Fedro psyché è principio del movimento e perciò immortale; nel Fedone psyché è animazione, principio vitale che, se si separa dal corpo, ne determina la morte. Psyché non a caso deriva da psychō, che riconduce etimologicamente al soffio, il respiro che è principio vitale, e allo stesso modo anima deriva da ánemos, il vento. Il termine greco psyché traduce anche la parola farfalla che compare nel racconto di Jensen, e a cui tra l'altro fa riferimento anche

I4. Per una lettura dell'interpretazione freudiana della ninfa in relazione alla concezione warburghiana si veda R. Kirchmayr, L'enigma della Ninfa, da Warburg a Freud. Un’ipotesi in due sequenze, "La Rivista di Engramma», I0o, settembre-ottobre 20I2, pp. I37-I53.

15. Per la trasformazione culturale della donna in ninfa dalla prima alla seconda metà del XV secolo nel contesto della signoria medicea e l'influenza del neoplatonismo si veda G. Lazzi, P. Ventrone, Simonetta Vespucci: la nascita della Venere fiorentina, Firenze, Edizioni Polistampa, 2007; riguardo alle caratteristiche della ninfa-anima nella filosofia antica cfr. S. Mati, Ninfa in labirinto. Epifanie di una divinità in fuga, Bergamo, Moretti e Vitali, 2006. 
Warburg, nel carteggio, paragonandosi a un entomologo. E un entomologo è significativamente il padre di Zoe/Gradiva.

Il legame con la fantasia e l'immaginazione è carattere proprio dell'anima secondo Aristotele. Nel De anima, la competenza dell'anima senziente per tutto ciò che è sensibile è tale da legarla all'immaginazione; nel De memoria et reminiscentia, la memoria, affezione dell'anima legata al tempo e che si dà attraverso l'immagine, è un phántasma, ossia un'affezione della sensazione, del pathos, quindi un patire dell'anima che genera immaginazione. Anche per Platone la memoria ha sede nell'anima, custode della conoscenza vera, per cui sapere è ricordare, l'anamnesi di una conoscenza tramandata attraverso i corpi per metempsicosi. I sogni, nella veglia come nel sonno, sono per Aristotele alterazioni dell'anima, in quanto immagini derivate dal movimento delle affezioni sensibili che hanno sede nell'anima. E l'immaginazione che si fa delirio, perdita di ragione, soprattutto nella forma della possessione, trova nella definizione di nymphóleptos il suo legame con la ninfa. La possessione di chi è catturato dalle ninfe, oltre a generare felicità, è anzitutto una forma primaria di conoscenza che trasforma chi ne è soggetto, quindi è una conoscenza subìta e metamorfica, che avviene attraverso il pathos. Un esempio tra tutti di nymphóleptos affine ai casi di Jolles e Jensen è quello di Socrate nel Fedro, dove l'oggetto del dialogo è una forma suprema di possessione, quella amorosa, la manía erotiké.

Nel neoplatonismo rinascimentale, il nesso anima-ninfa è colto da Marsilio Ficino come copula mundi, quindi nella funzione mediana di tramite tra sensibile e intellegibile, di riflesso del molteplice sensibile, e immagine del mondo, per cui le ninfe sono eídöla del mondo che fanno da tramite per gli eidōla della mente; l'anima-ninfa è l'Anima mundi in cui è riscattata la negatività della sua discesa nei corpi, nella materia. Ed è Eros, amore, il principio creativo che, come scrive Ficino rileggendo il Simposio e il Fedro, infiamma l'anima-ninfa permettendone l'epistrophé, il ritorno agli Intellegibili attraverso il piacere e la bellezza mondana.

\section{Ninfa come sintomo}

Gli aspetti tratti dalla tradizione filosofica antica illuminano la natura classica e pagana della ninfa-Gradiva descritta da Jolles e Jensen, e spiegano anche l'eccentricità con cui la figura torna in primo piano agli inizi del Novecento.

La ninfa, proiezione del mito e incarnazione figurata dell'anima, cattura l'attenzione della psicoanalisi che, con Freud, si appropria della sua 
immagine al punto da determinare la fortuna della novella di Jensen. Allo stesso modo la ninfa è l'immagine che guida al recupero e alla comprensione del mito nel contesto della scienza della cultura che Jolles elaborerà poi nella fase più matura della sua ricerca, con l'individuazione delle "forme semplici» (einfache Formen) dell'arte e letteratura. In entrambi i casi la ninfa è tramite alla comprensione e al valore del pensiero classico e mitico, un pensiero storicamente esiliato o rimosso dalla cultura dominante occidentale, divisa tra fede cristiana e culto secolare della ragione. Perciò la ninfa torna in epoca contemporanea come figura sintomale, secondo la nota definizione di Georges Didi-Huberman, che la intende come figura patologica proprio perché carica di contenuto rimosso, e nella fattispecie di pathos.

L'interesse di Freud per la Gradiva di Jensen ha un valore che va anche al di là della singola interpretazione, ed è indicativo anzitutto della funzione che la psicoanalisi, pur nella varietà dei suoi discorsi, ha avuto nel contesto della cultura scientifica e umanistica del Novecento per il recupero del pensiero mitico. Del mito viene riconosciuto il valore espressivo e gnoseologico, come capacità di dare immagini e parole al pathos, le passioni dell'anima che il linguaggio clinico ottocentesco non era in grado di descrivere e comprendere pienamente. Per questo Freud giustifica l'uso della «rappresentazione (Darstellung) particolareggiata dei processi psichici, quale in genere è data dagli scrittori» nella descrizione dell'isteria piuttosto che la "diagnostica locale e le reazioni elettriche» ${ }^{16}$. La psicoanalisi riscopre e riutilizza trame, nomi, volti antichi per dare senso all'esistenza quotidiana dell'uomo contemporaneo, e spiegare la psicologia individuale e collettiva dell'umanità. L'operazione inaugurata da Freud ha un valore rivoluzionario perché non solo restituisce alla narrazione mitica un valore di verità in grado di illuminare il presente storico dell'esistenza, ma agisce in ambito scientifico positivista, introducendo in esso la dimensione della poesia, del mito, della narrazione letteraria, e con essi del fittizio. La psicoanalisi mette quindi in discussione il confine posto dalle scienze positive per fondare se stesse tra la dimensione considerata veritiera dell'oggettivo e quella fittizia dell'immaginario. Come ha osservato Cesare Musatti, il saggio di Freud su Jensen è il primo caso in cui le categorie interpretative della psicoanalisi vengono applicate a un'esperienza narrata in un'opera letteraria e trattata come fatto reale ${ }^{17}$. Freud ne fa un fatto

I6. S. Freud, Studi sull'isteria, trad. it. e cura di C. L. Musatti, Torino, Bollati Boringhieri, 1989, p. 313.

17. Cfr. C. L. Musatti, Introduzione a Gradiva. Un racconto di Wilhelm Jensen e uno studio analitico di Sigmund Freud, in S. Freud, Saggi sull'arte, la letteratura e il linguaggio, cit., vol. 2, pp. 7-I7. 
reale al punto da considerare il protagonista della novella come un caso clinico esemplare, di manifestazione patologica dell'inconscio personale che trova sfogo nel delirio come conseguenza della rimozione di pulsioni erotiche provate nella prima infanzia. La visione del bassorilievo da parte di Norbert ha l'effetto di risvegliarne il rimosso perché assomiglia alla fanciulla che è stata oggetto del desiderio infantile represso.

Se Freud ha reintrodotto nel pensiero contemporaneo il valore vitale del mito, non ne ha evidenziato a sufficienza il valore collettivo e culturale, mantenendone vivo il significato simbolico e immaginale. Il caso di Gradiva è esemplare. Il mito è utilizzato alla fine come allegoria efficace; il linguaggio dell'inconscio deve essere smascherato dietro le trame del mito e tradotto dalla ragione interpretante come primitivo, legato a una patologica e irrisolta conflittualità sessuale infantile. In ultima analisi Freud, traducendo l'immaginale patologico nel reale, riduce la dimensione simbolico-immaginale del mito a espressione di uno stato psicopatologico ricorrente nell'uomo, e la colloca nell'inconscio personale. Così la vicenda raccontata nella Gradiva, in cui il delirio viene curato attraverso la scoperta della verità, ovvero del motivo che ha causato il disturbo patologico, simboleggia la funzione stessa della psicoanalisi ${ }^{18}$ come la intende Freud, ossia come traduzione dell'immaginale in reale. E la Gradiva sarà poi sostituita per importanza dal mito maschile di Edipo che diventerà fulcro della teoria freudiana ${ }^{19}$.

La ninfa-Gradiva ha un valore sintomale, è indice di un disturbo psichico perché esprime la repressione di una parte della vita pulsionale e la rimozione delle forme di rappresentazione attraverso cui l'impulso represso giunge a espressione; e tuttavia questo valore sintomale deve essere riconosciuto a livello non tanto o non esclusivamente personale, ma collettivo, compreso quindi nello studio più vasto della cultura. Questo è stato fatto dagli eredi di Freud, che hanno privilegiato lo studio dell'inconscio come immaginale e riconosciuto nella forma del pensiero mitico il linguaggio proprio della psiche, composto per l'appunto di immagini: un pensiero di pari dignità e valore rispetto alla forma del pensiero logico-razionale, che per le sue stesse caratteristiche non necessita di essere «tradotto». Se Carl Gustav Jung ha teorizzato gli archetipi dell'inconscio collettivo, dal canto suo James Hillman, concependo la «base poetica della

I8. S. Freud, Delirio e sogni della "Gradiva» di Wilhelm Jensen, ivi, p. 202.

19. Cfr. G. Huber, Warburgs Ninfa, Freuds Gradiva und ihre Metamorphose bei Masson, in S. Baumgart, G. Birkle (Hg.), Denkräume zwischen Kunst und Wissenschaft, Berlin, Dietrich Reimer Verlag, I993, pp. $443-460$. 
mente», ha riconosciuto come mito dell'analisi la favola di Eros e Psyche, dove psiche è l'Anima mundi di ascendenza neoplatonico-rinascimentale. Hillman pensa il Rinascimento come modello ideale di cultura vivificata dalla ricchezza immaginale della classicità, a cui la cultura contemporanea deve psicologicamente ricondursi. Perciò l'Italia e Firenze, cuore pulsante del Rinascimento, sono il luogo storico, geografico, simbolico a cui l'itinerario interiore dell'anima deve ricondursi ${ }^{20}$ : lo stesso luogo simbolico che il protagonista della novella di Jensen sperimenta nel viaggio in Italia all'inseguimento della Gradiva.

Jung ha affermato che gli «dei sono diventati malattie» ${ }^{21}$ : questo può rendere conto dell'esilio della ninfa-dea pagana. La cacciata degli dei ha provocato la loro malattia, perché non si può negare una necessità della psiche, quella immaginale, se non al prezzo della loro trasformazione mostruosa in demoni. Quindi se da un lato è significativo l'interesse psicoanalitico per la ninfa, dall'altro occorre andare oltre l'interpretazione freudiana, e considerare il valore culturale della sua figura all'interno della tradizione classica occidentale. È curioso e sintomatico che la letteratura critica fiorita sulla Gradiva abbia da un lato voluto riconoscere in Jung il "qualcuno» che secondo Freud gli avrebbe suggerito la lettura della novella, e dall'altro cercato di dimostrare fin troppo dettagliatamente ${ }^{22}$ che l'anonimo personaggio non poteva essere Jung. Ed è curioso anche che a tale attribuzione si accompagni la convinzione che Freud abbia scritto il testo per compiacere Jung ${ }^{23}$. Almeno idealmente, una lettura non "personalistica» del caso di Gradiva avrebbe potuto essere condotta dagli eredi di Freud; in questo caso Gradiva, nella interpretazione della psicoanalisi condotta da Jung o Hillman, avrebbe raccontato «il percorso dalla percezione individuale (estetica) alla memoria storica sovrapersonale», come ha osservato Settis in riferimento all'epistolario Warburg-Jolles ${ }^{24}$.

20. D. Sacco, Tradizione classica e logos della psiche. Una lezione sull'importanza della tradizione classica nel pensiero contemporaneo: James Hillman, in M. Centanni (a cura di), L'originale assente. Introduzione allo studio della tradizione classica, Milano, Bruno Mondadori, 2005, pp. I55-I6I.

2I. C. G. Jung, Commento al segreto del fiore d'oro, in Studi sull'alchimia, trad. it. di M. A. Massimello, Torino, Bollati Boringhieri, 1970, p. 47.

22. Cfr. L. Russo, La nascita dell'estetica di Freud, Bologna, Il Mulino, I983, pp. I83-219.

23. Cfr. C. L. Musatti, Introduzione a Gradiva. Un racconto di Wilhelm Jensen e uno studio analitico di Sigmund Freud, cit.

24. S. Settis, Presentazione, in J. Seznec, La sopravvivenza degli antichi dei. Saggio sul ruolo della tradizione mitologica nella cultura e nell'arte rinascimentali, cit., pp. VII-XXIX. 


\section{Jolles e la riflessione sul mito}

Sulle stesse tracce dello studio sintomale - ma non riduttivamente individuale — delle forme del mito di cui la ninfa è espressione primaria si colloca la riflessione di Jolles, che, lo abbiamo visto, riconosce molto presto la necessità di fondare lo studio della cultura su basi psicologiche. La figura della ninfa, su cui si concentra nel carteggio e più tardi in un rinnovato interesse stimolato dallo studio sul Boccaccio (I92I), catalizza in nuce gli elementi che il critico olandese attribuisce al pensiero mitico. Pensiero che ha i suoi nuclei più forti non solo nelle Forme semplici (1930), ma anche nell'importante saggio intitolato Folklore e scienza dell'arte (I897) e nel carteggio su Clio e Melpomene con Johan Huizinga (1925).

In Folklore e scienza dell'arte, Jolles per "folklore» intende i miti e le fiabe. Come nota Silvia Contarini ${ }^{25}$, «scienza dell'arte» sostituisce «storia dell'arte», e prelude così alla novità di una riflessione nascente sulla disciplina. Nel confrontare le origini del metodo di Warburg a quelle della psicoanalisi, Didi-Huberman - che significativamente riconduce alla Gradiva anche il fenomeno fin de siècle dell' isteria, altra femminile espressione di pathos - afferma che, affinché nasca qualcosa che possa definirsi come moderna «scienza delle immagini», occorre «un'apparizione che con la stessa forza sappia rovesciare un'altra forma di sapere scolastico: la storia dell'arte» ${ }^{26}$. È la linfa vitale dischiusa dalla ninfa a sovvertire il sapere sterile (per la psicoanalisi incarnato nella scienza positivista), incapace di parlare al presente e rispondere alle nuove domande di senso. Anche per Norbert Hanold, sofferente per la scolasticità degli studi a cui si è votato e che lo hanno allontanato dalla vita, l'incontro con la ninfa-Gradiva rappresenta il contatto con la potenza vivificante in grado di riportarlo «a una conoscenza che coinvolgesse lo spirito, l'anima, il cuore».

Per quanto appartenga agli anni di formazione, il testo sul folklore di Jolles non sarà mai considerato superato dal suo autore. Anzi, alla vigilia dell'edizione di Forme semplici verrà riconosciuto come depositario di «questioni» e «ipotesi fondamentali» ${ }^{27}$. In questo saggio Jolles pone infatti un problema non trascurabile relativo al mito, svelandone l'aspetto sintomale:

25. Cfr. S. Contarini, Tra folklore e scienza dell'arte, cit., pp. I53-I72.

26. G. Didi-Huberman, Ninfa moderna. Saggio sul panneggio caduto, trad. it. di A. Pino, Milano, Il Saggiatore, 2004 (prima edizione italiana 2002).

27. Cfr. E. Raimondi, Premessa, in S. Contarini (a cura di), André Jolles. I travestimenti della letteratura, cit., pp. V-VIII. 
[...] se il folklore è simile a una manifestazione patologica, una perla che nasce dalla malattia dell'ostrica e tuttavia si conserva a lungo come ornamento dopo la scomparsa dell'organismo, dove si trova, dentro di noi, il granello di sabbia che provoca la preziosa crescita? ${ }^{28}$

Oltre a rivelare il valore sintomale del mito, la domanda di Jolles rappresenta la premessa da cui partire per comprendere la genesi delle forme culturali. Ciò che interessa qui non è tanto definire l'essenza del mito, perché il compito dello studioso, coerentemente a una prospettiva morfologica che non presuppone una sorta di sostanzialità conoscibile dell'oggetto, consiste nel comprendere come una data domanda nell'uomo abbia potuto generare una corrispondente risposta. La richiesta di senso sta proprio nell' «accordo tra l'esterno e quel che è all'interno" invocato da Socrate nella preghiera a Pan e alle ninfe in chiusura del Fedro (279b). Nell'accordo o disaccordo, connessione o non-connessione si colloca anche il rapporto che secondo Jolles esiste tra la domanda e la risposta. È nel rapporto di vicinanza o distanza tra l'idea e l'immagine, riflesso nel rapporto tra la domanda e la risposta, che si comprende storicamente la genesi delle forme della cultura; l'immagine risponde all'idea, e la forma peculiare del mito è significativamente proprio la «trasformazione in immagine» ${ }^{29}$. Ma la dimensione immaginale è propria del mito, come per la psicoanalisi è propria dell'inconscio; trascende il caso specifico della realtà ed è sottratta alle leggi della storia, perciò è la dimensione universale all'interno della quale si comprende il mito. L'universale di pertinenza del mito, della poesia, e il particolare di pertinenza della storia, come nella teoria aristotelica, per Jolles devono essere tenuti assieme come le due facce di una stessa medaglia. La comprensione dell'immagine al di fuori del tempo e dello spazio ne spiega l'universalità senza negarne la variazione, il suo essere metamorfico. Il mito, afferma Jolles, «coglie proprio ciò che è universale ma costante nella sua molteplicità ${ }^{30}$. L'immagine può mutare nel tempo, subire delle metamorfosi, coerentemente alla teoria goethiana della forma, e quindi può non corrispondere più alla domanda, all'idea per cui si era generata. Perciò il mito è la soddisfazione di una fantasia, della domanda di un'immagine, al contrario della domanda di chiarimento razionale propria dell'uomo civilizzato ${ }^{31}$. E significativamente, in perfetto accordo con le teorie della psicoanalisi, il processo di trasformazione in

28. A. Jolles, Folklore e scienza dell'arte, cit., p. I9.

29. Ivi, p. I8.

30. A. Jolles, Forme semplici, cit., p. 337.

3I. A. Jolles, Folklore e scienza dell'arte, cit., p. I3. 
immagine proprio della creazione del mito ha, secondo Jolles, un importante corrispettivo nel «sogno», che «rappresenta un mezzo prezioso per osservare la metamorfosi delle impressioni in immagini dentro di noi» ${ }^{32}$. Come nel mito, nel sogno si verifica un'universalità delle forme data dalla combinazione "fuori dallo spazio e dal tempo" degli elementi che lo compongono; perciò il sogno è un modello di riferimento per lo studio del mito, come per la psicoanalisi il mito è un modello di riferimento per lo studio del sogno. Portare il sogno dentro la scienza è dunque ciò che accomuna la nascente scienza dell'arte alla psicoanalisi. Nella novella di Jensen, scienza e sogno sono le due forze che, per quanto mosse fino allora su fronti contrapposti, contribuiscono di pari passo allo stato in cui si trova il protagonista nell'inseguimento della ninfa-Gradiva ${ }^{33}$. Non per nulla, Norbert Hanold riconosce che è «quella condizione dell'anima che chiamiamo sogno» a portarlo indietro nel tempo, a Pompei.

Sul significato della domanda, Jolles tornerà ulteriormente nel libro della maturità, dove il Mito è studiato come specifica "forma semplice» e inteso come portato di desiderio. La domanda consiste nel desiderio di ottenere una risposta; in quanto tale anticipa la richiesta di conoscenza, e si chiarifica ancora una volta come richiesta di senso. Il desiderio è pure desiderio estetico, ossia un «desiderio di bellezza» che va "anche al di fuori dei confini propri dell'arte» per comprendersi nel più vasto desiderio culturale. Perciò il desiderio ha la sua incarnazione nella ninfa ${ }^{34}$; la ninfa è figura del desiderio di bellezza, come nella tradizione classica: la caduta dell'anima, la discesa nel mondo è dettata dal desiderio di creare (Corpus Herm., I, 4), che la attira verso il mondo terreno. Significativamente, nelle Forme semplici, «la creazione» è l'ulteriore elemento che Jolles attribuisce al mito: è propriamente sullo stimolo del desiderio della domanda che si dà creazione nella risposta. Il Mito è «la forma capace di creare oggetti a partire da una domanda e da una risposta», e non può che essere forma vitale, perché la domanda è sempre pensata come legata a una «disposizione mentale» ${ }^{35}$ e perciò compresa nella sua dimensione psicologica.

$\mathrm{La}$ «manifestazione patologica del folklore» è una conseguenza del fatto di considerare "errori» forme di cultura di cui non si riconosce più la domanda originaria; sono risposte che non appartengono più alla cultura in cui sussistono, e ciò dipende dal fatto che "la civiltà del pensiero ha

32. Ivi, p. 20.

33. Cfr. W. Jensen, Gradiva, una fantasia pompeiana, cit., p. 35.

34. Cfr. G. Didi-Huberman, Ninfa fluida. Essai sur le drapé-désir, Paris, Gallimard, 20I5.

35. A. Jolles, Forme semplici, cit., p. 328. 
respinto l'attività di formazione delle favole al punto che non è rimasto alcuno spazio per essa nella nostra vita spirituale» ${ }^{36}$. A questa convinzione si accompagna il dubbio che lo stato primitivo a cui si riconduce solitamente il Mito sia uno "stato di decadenza o di degradazione», o che il popolo selvaggio possegga «una capacità psichica» diversa dal popolo civilizzato. Persuaso che «uno dei primi compiti del nuovo secolo sarà di ricostruire una sorta "di storia universale delle origini» ${ }^{37}$, e prefigurando quindi il Novecento come secolo in cui torna viva l'attenzione per quelle che ha nominato "forme semplici», Jolles è consapevole dei limiti delle scienze nel tentativo tardo-ottocentesco di spiegare il mito attraverso il metodo linguistico, quello etno-psicologico e quello analogico. Il filtro razionale che questi approcci utilizzano per la comprensione del mito nega la considerazione del portato di pathos che gli dà forma. Il coefficiente emotivo della forma, la natura «vivente» delle "forme semplici» espressa nella vitalità della loro continua mutazione trova la sua cifra espressiva nel movimento. Le "sopravvivenze dell'Antico», ovvero i frammenti che vengono dal passato, devono essere considerate per il portato di vitalità, il valore di senso che hanno avuto non solo per la cultura in cui si sono formate, ma anche per quella che, in un altro tempo e spazio, le comprende. Il fossile quindi, come la pietra del bassorilievo che ritrae la ninfa-Gradiva, va considerato per la vitalità che è ancora in grado di esprimere dentro la forma. Coerentemente all'affermazione di Jolles contenuta in una lettera a Huizinga secondo cui «il Rinascimento ebbe la sua culla in una tomba» ${ }^{38}$, il fossile, che racchiude in sé un'idea di morte, è segno di vita. La concezione di sopravvivenza ha a che fare con la sua vita spettrale, fantasmatica; l'antico bassorilievo della ninfa-Gradiva agli occhi del protagonista è «un monumento funebre» con cui lo scultore aveva tramandato alla posterità l'immagine della fanciulla precocemente strappata alla vita. La rianimazione del bassorilievo restituisce alla figura una vita fantasmatica, nell'oscillare continuo tra la dimensione della realtà e della fantasia. Realtà e fantasia, mito e conoscenza sono per Jolles legati da attrazione e repulsione: una lotta continua tra la «disposizione mentale» che produce il Mito e la volontà di conoscenza che illumina il reale. In questa dialettica si può forse leggere la relazione tra Clio e Melpomene che, nel carteggio con Huizinga, è espressa nella domanda aperta di Jolles se «Clio non sia in

36. A. Jolles, Folklore e scienza dell'arte, cit., p. I9.

37. Ivi, p. 5 .

38. La citazione di Jolles è tratta da S. Contarini, Un dialogo epistolare sul Boccaccio: Jolles, Huizinga e il "problema del Rinascimento", "Studi sul Boccaccio», 39, 20II, pp. 217-24I. 
se stessa una sorta di Melpomene», se "la "storia" stessa non sia una sorta di "poesia" che non conosce in senso stretto alcuna forma letteraria, ma non può fare a meno di servirsi delle forme semplici»" ${ }^{39}$. A guardare bene, è un'affermazione simile a quella espressa da Hillman in Edipo rivisitato ${ }^{40}$, secondo il quale il compito della psicoanalisi non deve essere tanto la traduzione della fantasia in realtà, dell'immaginale in letterale, ossia il percorso indicato da Freud, quanto l'osservazione del reale attraverso la fantasia.

In tale prospettiva, che sembra di poter attribuire in parte anche a Jolles, i termini «reale», "letterale», «fantastico», «immaginale» si conservano, ma il loro ruolo nella comprensione dell'uomo e del mondo è rovesciato. Attraverso il mosaico dei testi citati fin qui si comprende allora meglio la sopravvivenza, lungo il Novecento, del simbolico atto di purificazione che compie Socrate nel Fedro prima di intraprendere il discorso vero su Eros, rivolgendosi a Pan e alle ninfe. Come fa notare Calasso, esso equivale a un atto riparatore verso una potenza offesa per aver peccato nei confronti di Eros e della mitologia, e per aver misconosciuto la lingua dei simulacri: una "sapienza che parla per gesti e per immagini»; e quest'atto è rivolto, oltre che a Pan, alle ninfe, in quanto mediatrici di Eros e della mitologia ${ }^{41}$.

39. A. Jolles, Clio e Melpomene, in S. Contarini (a cura di), André Jolles. I travestimenti della letteratura, cit., p. 250. Per un inquadramento del carteggio cfr. l'introduzione di S. Contarini, in A. Jolles, J. Huizinga, Clio et Melpomène, «Poétique», Ioo, 1994, pp. 487-488.

40. Cfr. J. Hillman, Edipo rivisitato, in K. Kerényi, J. Hillman, Variazioni su Edipo, trad. it. di T. Cavallo, A. Serra, Milano, Raffaello Cortina, I992, pp. 75-I43.

4I. R. Calasso, La follia che viene dalle Ninfe, Milano, Adelphi, 2005, pp. 35-36. 